\title{
Three Levels of Cognition: Particulars, Universals, and Representals
}

\author{
Nijalingappa Umakantha \\ Department of Physics, Karnatak University, Dharwad, India \\ Email: numakantha131@yahoo.co.in
}

How to cite this paper: Umakantha, N. (2016). Three Levels of Cognition: Particulars, Universals, and Representals. Open Journal of Philosophy, 6, 335-345.

http://dx.doi.org/10.4236/ojpp.2016.64033

Received: July 4, 2016

Accepted: October 10, 2016

Published: October 13, 2016

Copyright (๑) 2016 by author and Scientific Research Publishing Inc. This work is licensed under the Creative Commons Attribution International License (CC BY 4.0).

http://creativecommons.org/licenses/by/4.0/ (c) (i) Open Access

\section{Abstract}

It is shown that apart from the two well known levels of cognition involving the epistemological concepts particulars and universals, there is an intermediate level of cognition necessitating a new epistemological concept which we call represental. This has become necessary as a result of emergence of statistics (an empirical science), the theory of probability (a branch of pure mathematics), and quantum mechanics (as a branch of physics) at the beginning of the nineteenth century. We attribute to a particular man (like Mr. Jones) well defined properties (like a definite number of children) whereas we attribute to a man, the universal, only some general properties (like having an erect body). Thus particulars and universals involve two levels of cognition. In statistics, we deal with the properties of a large number of particulars denoted by a universal, without referring to such details as which particular has which properties. Thus statistics involves a new level of cognition. In statistics, we attribute all the statistical properties to a single entity and refer to it as the represental (entity); the concept of represental man is only a generalization of the concept of average man proposed by Quetelet in 1869 . These three epistemological concepts are distinguished by the relation they bear with respect to the possible "states" of the particulars. For instance, Mr. Jones, a particular man, can be in the state of having either 0 , or 1 , or $2, \ldots$ children only; a man, the universal, cannot be said to have either 0 , or 1 , or $2, \ldots$ children, though the state of having children is relevant to him (but not to a chair, the universal); the represental man has 0 child with probability $P(0), 1$ child with probability $P(1), 2$ children with probability $P(2), \ldots$ Thus the possible states are mutually exclusive in particulars, are only relevant to the universal, and coexist in the represental with respective probabilities. By recognizing that in statistics, the theory of probability, and quantum mechanics we deal with a new level of cognition involving the epistemological concept of represental, the interpretational problems of statistical phenomena are resolved. 


\section{Keywords}

Three Levels of Cognition, Particulars, Universals, Representals, Monadic and Collectivistic Probabilites

\section{Introduction}

From the days of Plato the problem of denoting a large number of particulars (objects of perception) belonging to a kind by a universal (an object of thought) has been a perennial subject of discussion among epistemologists, Moreland (2001) but of little interest to scientists. It is only at the beginning of the nineteenth century that scientists began to study the average properties of a large number of particulars denoted by a universal. This leads to development of statistics as a new branch of knowledge (Kendall, 1979). During the middle of the seventeenth century, the theory of probability was developed with reference to the games of chance with equipossible outcomes but emerged as a branch of pure mathematics only in early nineteenth century. That statistics and probability theory are closely related disciplines was recognized only at the beginning of the twentieth century (Spigel, 1962; Jaynes, 2003). During the same period quantum mechanics, based on probability, emerged as a new branch of physics (Home \& Whitaker, 1992). Though these three disciplines are well developed from the point of mathematical rigor and range of applications, their basic concept, namely probability, has remained a subject of much discussion regarding its meaning. Even today there is no universally accepted definition of probability (Howson, 1995). The main reason for this is the inadequacy in epistemology in not recognizing that statistics, probability theory, and quantum mechanics belong to a new (intermediate) level of cognition distinct from the two well known levels of cognition which involve the epistemological concepts particulars and universals.

In statistics, we do not take cognizance of such details as the space-time regions of the particulars and which particular has which specific properties, but are concerned with the average values and the distribution of values; these statistical properties are independent of the actual number of particulars of a population and so are expressed with reference to a single purely conceptual object, called the average entity. The concept of average man was first introduced into statistics by Quetelet (Quetelet, 1869). Whereas a particular man (like Mr. Jones) can have either 0 , or 1 , or $2, \ldots$ children, the average man can have 2.54 number of children. Evidently the average man is neither a particular man nor a universal man. Thus we recognize that this new discipline of statistics involves a new level of cognition which is distinct from the two familiar levels of cognition relevant to particulars and universals. We generalize the concept of average man and call it the represental man. The distinctive features of these three epistemological concepts are: a particular man (like Mr. Jones) can have either 0 , or, 1 or $2 \ldots$ children; we cannot attribute to a man, the universal, either 0 , or 1 , or $2, \ldots$ children; we attribute to the represental man 0 , and 1 , and $2, \ldots$ children with respective proba- 
bilities (whose sum is exactly unity). Recognition that statistics, the theory of probability, and quantum mechanics belong to a new level of cognition solves the much debated interpretational problems of both probability theory and quantum mechanics. First we mention the main features of particulars and of universals and then discuss those of representals.

\section{Particulars}

It is our everyday experience that a multitude of individual objects of perception, referred to in philosophy as particulars, exist in nature. All our experiences and (experimental) observations involve particulars only. According to Thomas Aquinas a particular is defined by its spatiotemporal position and according to Leibniz a particular is constituted by qualities (Russell, 1948). Thus every particular is characterized by a definite space-time region and some definite qualities, or properties, or attributes. Rather than considering such complex entities as mice and men, we may consider more elementary objects like triangles and electrons.

Let us consider the properties of an object obtained by bending a thin iron wire into the shape of a triangle formed by the straight sides $\mathrm{AB}, \mathrm{BC}$, and $\mathrm{CA}$ having definite lengths, placed on a table at some definite location. It has many properties such as: the sum of the three internal angles is $2 \pi$; the sum of any two sides is greater than the third; the lines bisecting the angles at $\mathrm{A}, \mathrm{B}$, and $\mathrm{C}$ meet at a point; the perpendicular bisectors of the sides $\mathrm{AB}, \mathrm{BC}$, and $\mathrm{CA}$ meet at a point; a unique circle which just touches the lines $\mathrm{AB}, \mathrm{BC}$, and $\mathrm{CA}$ each at one point, exists; a unique circle which passes through all the three points $\mathrm{A}, \mathrm{B}$, and $\mathrm{C}$, exists; etc. The important point to notice is that once the lengths of the three sides are fixed, all the properties of the triangle follow from the laws of geometry. So we recognize the three straight lines as the basic property of the triangle. As it has a definite space region and well defined properties, the triangle is a particular (entity).

Next, let us consider what is probably the most elementary entity in nature, the electron. Consider a single particular electron adhering to an oil drop in some define location. The electron has the following properties: mass, electric charge, spin angular momentum, and magnetic moment, each having a well defined numerical value, independent of the space-time region of the electron; these are called the intrinsic properties. The electron also has other properties such as velocity, linear momentum, kinetic energy, etc. whose values may change with time. These are called the extrinsic properties.

The important point to notice is that the intrinsic properties are the basic properties which identify an entity as an electron and the extrinsic properties specify the "state" of the electron at an instant of time (as governed by the laws of physics). The intrinsic properties together with the space-time region identify the entity as a particular electron.

\section{Universals}

Let us think of a triangle $A B C$ without specifying the material and the diameter of the 
wire, the space-time region, and the lengths of the three sides. All the properties of this triangle are interrelated according to the laws of geometry (just as in the case of the particular triangle) but their numerical values remain unspecified. Such a triangle is an object of thought. We may refer to it as the universal triangle. More conveniently, we may refer to it as a triangle. In popular parlance, we refer to the universal triangle as a triangle and refer to any particular triangle as the triangle. (We may mention that the indefinite and definite articles $a$ and the do not exist in many languages of the World, including the classical language Sanskrit; in fact they did not exist in old English either and were later borrowed from the classical language Greek.)

Similarly, we can think of an entity to which we attribute the intrinsic properties of the electron but keep the numerical values of the extrinsic properties and the space-time region unspecified. Such an entity is an object of thought. We may refer to it as the universal electron. More conveniently, we may refer to it as an electron. In popular parlance, we refer to the universal electron as an electron and refer to any particular electron as the electron.

\section{Many Particulars of a Kind and a Single Universal}

If there is to be a universal there should necessarily be many particulars which satisfy two basic conditions: i) all of them have some basic properties in common so that they can be regarded as belonging to a kind or to a reference class, and ii) they should differ in some other properties (at least in their space-time regions) so that each can be regarded as a particular. We may consider some typical cases.

Case 1. In the extreme case of freshly minted coins (or printed postage stamps) of the same denomination, all of the coins (or stamps) have the same properties but differ only in their space regions; they are identical entities and belong to a kind denoted by the universal, a coin (or a stamp).

Case 2. In the case of many particular triangles each is specified by: a definite space region, the material and the diameter of the wire, and a set of three straight lines of definite lengths. Though the lengths of these lines differ from triangle to triangle, all the triangles have the same set of properties expressible in terms of these lengths as determined by the laws of geometry. The triangles belong to a kind denoted by the universal a triangle.

It is possible to consider some of these particular triangles as belonging to a smaller reference class by recognizing some special properties common to them. For instance, all the triangles having two sides of equal length (but their magnitudes being unspecified), are said to belong to a reference class denoted by the universal, an isosceles triangle. Similarly, we can have the universals: an equilateral triangle, a right-angled triangle, an isosceles right-angled triangle, etc. Evidently, a particular triangle can be a member of more than one reference class. Thus depending on the properties we choose as the basic properties for considering a large number of particulars as belonging to a reference class, we get different universals. A universal is meaningful only with reference to a well defined (?) reference class. 
Case 3. In the case of many particular electrons, the electrons exist within different space-time regions and have the same intrinsic properties. They are regarded as identical entities belonging to a kind denoted by the universal an electron; needless to state that at any instant of time they may have different sets of extrinsic properties.

In all these three cases the basic properties of the particulars which belong to a reference class can be specified accurately. In the case of more complex entities in nature (like plants and animals) the properties attributable to the particulars are so numerous and vague that it is often difficult to specify verbally the basic properties relevant to (what we wish to regard as) a reference class. But, it is well known that every animal has inherent ability to recognize whether the other animal in its vicinity is harmful or not, indicating that there is some biological basis for particulars and universals

It is a fact of life that many individual animals are born, grow into adulthood, reproduce, and die; each animal retains its identity by existing at every instant of time within a gross region of space. But the survival of every species depends on the ability of the members of the species: i) to recognize their food (available in the form of edible vegetation or animals); ii) to escape from their predators; and iii) to recognize the members of its own species but of opposite sex. This shows that every individual animal has the ability to recognize whether the animal in its proximity belongs to its own kind, or to a harmless kind, or is a predator.

Similarly, it is a fact of life that many individual men are born, grow into adulthood, reproduce, and die; each individual retains his identity by existing at every instant of time within a gross region of space. But, for survival even in a primitive society every man should retain his identity as a particular man through ownership of property, his kinship with other members of his family and his tribe, division of labor, etc.; in a more advanced society he should also have citizenship identity, a bank account, a driving license, etc. No man can survive in a society, primitive or otherwise, without recognizing scores of other men as particular men and without being recognized as a particular man by scores of other men.

Thus recognition of universality is an instinctual necessity for survival of animals and recognition of particularity is a social necessity for survival of human beings. Being both an animal and a human being, every individual man has instinctual ability to recognize the universal in a particular and an acquired ability to recognize the particular in a universal. This is evident when a tourist visits for the first time a country inhabited by people of a different race. He can readily recognize that the different particular men belong to the same race but cannot often identify a particular man (of the race) as the one who has been introduced to him earlier. But he can readily distinguish any two identical twin-brothers of his own race, however close is their identity.

\section{Statistics, a New Branch of Knowledge}

"The broad river of thought that today is known as theoretical statistics cannot be traced back to a single source springing identifiably from the rock. Rather it is the confluence, over two centuries, of a number of tributary streams from many different re- 
gions... At the end of the nineteenth century "statistics" came to mean any numerical material that arose in observation of the external world" (Kendall, 1979). More explicitly, statistics deals with quantitative data relevant to the properties of a large number of particulars which belong to a reference class denoted by a universal. For the sake of clarity we may consider adult men in a population. Every man, like Mr. Jones, is a particular and has an identifiable space region in the form of his place of residence and has several properties attributable to him many of which (like age, height, weight, marital status, number of children, etc.) can be expressed quantitatively. Evidently, the (numerical) values of these properties differ from person to person. Statistics deals with this type of data relevant to a large number of particulars.

In order to make clear the main features of statistics we just consider one property, namely the number of children. Let us define the "state of parenthood" as the number of children an adult man has and denote the possible states by $C_{0}, C_{1}, \ldots, C_{5}$, corresponding respectively to the number of children being $0,1, \ldots, 5$; for the sake of simplicity we have limited the maximum number to 5 . Evidently, any particular man (like Mr. Jones) exists, over a duration of time, in only one of the possible states $C_{0}$, or $C_{1}, \ldots$, or $C_{5}$.

We may collect the data about the states of parenthood of the adult men in a large population by approaching one particular man after the other in a systematic manner (by going from house to house, street to street, and city to city) and record the states in a sequence. If in a long segment of this sequence containing $N$ number of entries, the state $C_{2}$ occurs $N_{2}$ number of times, the quantity $\omega(2)=N_{2} / N$ is called the relative frequency of the state $C_{2}$ in the segment; this relative frequency is just the fraction of the total number of men considered in the segment who are in the state $C_{2}$. Similarly we can have the relative frequencies $\omega(0), \omega(1), \ldots \omega(5)$; needless to state that the sum of these six relative frequencies is identically one. It is found that if $N$ is sufficiently large, of the order of 10,000 or more, each state has approximately a constant relative frequency independent of the actual number $N$ in the segment and also independent of from which part of the sequence the segment is chosen. These relative frequencies are also independent of such details as which particular man resides in which particular house and has which particular state of parenthood.

Further, there is no unique way of selecting city after city, street after street, and house after house for collecting the data. Thus we can have many different sequences of the same states $C_{0}, C_{1}, \ldots, C_{5}$ belonging to the same population. An important empirical fact which makes statistical analysis meaningful at all (as a general method) is that all these sequences lead approximately to the same set of values of relative frequencies, indicating that these relative frequencies are characteristic of the population. Or in other words, the percentages of men having different numbers of children are the same over all parts of the geographical area of the population. A little reflection would show that this is a direct consequence of the absence of any relation between the places of residence and the states of parenthood of the particular men in the population; we may recall that these are (according to Thomas Aquinas and Leibnitz) the two basic properties which identify the particulars. 
An analysis of the data in a sequence in which (analysis) no cognizance is taken of which possible state belongs to which particular entities, is called statistical analysis.

\section{A New Level of Cognition and the Represental}

In statistical analysis we deal only with the possible "states" and their relative frequencies, without making references to the individual particular men in the population. Now the most crucial epistemological question is: if the states $C_{0}, C_{1}, \ldots, C_{5}$ are free from references to the particular men (like Mr. Jones, Mr. Smith and others) in the population, then whose states do they denote in statistical analysis? After all, a state (i.e., an attribute) cannot exist without some object (or an entity) possessing it. Grin cannot exist without the Cheshire cat!

In order to find a solution to this epistemological problem, let us consider the typical statistical quantity, namely, the average or the mean number of children "a man" in the population has. Being a statistical quantity, this mean number need not be an integer and can have a non-integral value, such as 2.72. Evidently, no particular man, like Mr. Jones, can have 2.72 children. We can only identify it as the number of children of the average man of the population. The concept of average man was, as mentioned earlier, first introduced into statistics by Quetelet during the mid-nineteenth century.

Historically, this concept was popular among statisticians for many decades but slowly waned. If the epistemological significance of this concept was recognized by the philosophers of science, much of the later controversy in interpreting probability and quantum mechanics would have been avoided (Umakantha, 2016).

As no particular man in the population can be the average man, the average man cannot be an object of perception and can only be an object of thought. A man, the universal, is also an object of thought but we cannot attribute to a man (the universal) 2.72 number of children. Thus the average man of statistics is a new kind of object of thought. Now we show that it belongs to a new level of cognition (which is distinct from the two well known levels of cognition which lead to the well known epistemological concepts, particulars and universals).

In identifying a particular man as Mr. Jones we recognize many well defined properties of Mr. Jones whereas in identifying many particular men as belonging to a kind denoted by the universal, a man, we recognize only certain basic features of these particular men. Here we adopt two extreme levels of cognition. On the other hand, in statistical analysis we are not interested in which particular man is in which particular state but are only interested in the fractions of the total number of particular men in the population who are in these different possible states; thus in statistical analysis we adopt an intermediate (between the particular and the universal) level of cognition. This is the reason why we have to invoke a new epistemological concept which is commensurate with this intermediate level of cognition. The concept of the average man belongs to this intermediate level of cognition.

The concept of average has limited connotation. In statistical analysis, apart from average values, we deal with distributions of possible values also. Further, in the case a 
property like the eye color of the men in a population (which may be black, blue, brown, green, grey, etc.), the distribution of eye color is meaningful, but the average eye color is meaningless. So, we need a more general concept than the average for developing a new method of dealing with the statistical data. As the words representative, representent, representer do not have the requisite connotation, we coin a new word, "represental". This word rhymes with the word universal and, like it, is a noun as well as an adjective.

We express all the statistical data relevant to a population of a large number of particulars with reference to a single entity called the represental (entity). For instance, with reference to the states of parenthood of the men in a population, we define the represental man as "one man" whose state of parenthood is $C_{0}$ to the relative extent $\omega(0)$, $C_{1}$ to the relative extent $\omega(1), \ldots$, and $\mathrm{C}_{5}$ to the relative extent $\omega(5)$. Or in other words, the represental man exists in all the possible states $C_{0}, C_{1}, \ldots, C_{5}$ to the relative extents $\omega(0), \omega(1), \ldots, \omega(5)$, respectively.

By way of clarification we may state that the concept of represental is distinct from the concept of class. A class of men is not a man, but the represental man is "one man". A flock of birds is not a bird, but the represental bird (defined with reference to some property such as weight) is "one bird".

\section{Three Levels of Cognition}

We have recognized that human knowledge involves three levels of cognition based on the three epistemological concepts, particulars, universals, and representals. Particulars are objects of perception on which human experience and knowledge depend. Universals are objects of thought which are necessary for forming general conclusions, for communication, and for expressing the laws of nature. Representals are objects of thought which have now become necessary for dealing with statistical data. All these three levels of cognition involve the same states but the status of the states are different in different levels of cognition.

Let us consider, for instance, the relations the possible states $C_{0}, C_{1}, \ldots, C_{5}$ bear with respect to a particular man, the universal man, and the represental man. Only one of the possible states of parenthood, either $C_{0}$, or $C_{1}, \ldots$, or $C_{5}$ can be attributed to a particular man. No definite state, neither $C_{0}$, nor $C_{1}, \ldots$, nor $C_{5}$, can be attributed to the universal man (though the concept of state of parenthood is relevant or meaningful with reference to the universal man). All the possible states, $C_{0}$, and $C_{1}, \ldots$, and $C_{5}$ are attributed to the represental man (to the relative extents $\omega(0), \omega(1), \ldots, \omega(5)$, respectively). That is, the possible states are mutually exclusive in a particular, are only relevant to the universal, and coexist in the represental.

\section{The Probability Theory and Statistics}

In the theory of probability (a branch of pure mathematics) we deal with statistical problems in an abstract and a mathematically more rigorous manner. In the theory of probability per se, the possible states are unidentified (in the sense that they are just 
denoted by symbols $S_{\mathrm{n}}$ 's) and the numerical values of the probabilities $P(\mathrm{n})$ 's are treated as unspecified constants. In applying the results of the theory to a given statistical data, the states are identified and $P(\mathrm{n})$ 's are estimated from the given statistical data (Umakantha, 2016).

Though both statistics and probability theory belong to the intermediate level of cognition, they are, from the epistemological point of view, two distinct disciplines. The particulars, mutual exclusion of the possible states in a particular, the sequences of states, randomness, and the relative frequencies of the states belong to the province of statistics whereas the represental, coexistence of the states in the represental, and the probabilities, belong to the province of probability theory. Only the possible states are common to both. Thus the concept of randomness is foreign to the probability theory and the concept of probability is foreign to statistics. Suffice it to observe that the relation between the two disciplines does not involve any epistemological issues.

\section{Quantum Mechanics and Probability Theory}

The concept of probability is the same in both probability theory and quantum mechanics. The theory (per se) of probability being a branch of pure mathematics, the concept of time does not play any explicit role in the theory; but the concept of time is an integral part of physics. So, quantum mechanics has some features not found in the general discussion on probability theory. Quantum mechanics deals with phenomena exhibited by atoms. In the simplest case, an electron (mentioned in Section 2) is bound to another entity called the proton; the two together form a composite entity (a hydrogen atom).

There are many discrete possible "states" for a hydrogen atom and a particular hydrogen atom exists, over a duration of time, in only one of the many possible states. Each state is specified by a unique value of energy, one well defined probability distribution for the position and another for the momentum of the electron. That the position of the electron has a probability distribution impliess that it has different values at different (well separated) particular instants of time leading to a random sequence and the probability distribution refers to the represental instant of time. This is so with the momentum of the electron also. These probability distributions which are relevant to a single atom are called monadic probabilities. It can be shown that monadic probabilities should necessarily be independent of time (Umakantha, 2016).

It is possible to subject a large number of atoms (or atomic systems), each initially in the same state, to some external physical conditions one after the other so that the successive atoms come to exist later in different possible states, leading to a random sequence. In this case also, the laws of quantum mechanics determine the probability distributions (without reference to any statistical data). The probability distributions which are relevant to a large number of atoms are called collectivistic probabilities.

The laws of quantum mechanics determine the possible states, the values of energy, and the monadic probability distributions relevant to single atoms; and the laws also determine the collectivistic probabilities relevant to a statistical assembly atoms (without reference to any statistical data) (Umakantha, 2016). 
From the point of epistemology, the following points may be worth deeper study: i) Our understanding of even what is probably the simplest composite entity in nature, namely a single particular hydrogen atom, involves all the three levels of cognition. ii) By explicitly introducing the concept of time into the theory of probability, we are able to distinguish between the monadic properties of single atomic systems and the collectivistic properties of a large number of atomic systems. iii) Whereas in the theory of probability per se the possible states and the numerical values of probabilities are unspecified, in quantum mechanics they are determined by the laws of physics. Does this mean that probability is rooted in physical phenomena rather than in statistical data?

\section{A Few General Remarks}

The following observations may be of interest to students of philosophy.

1) Logically speaking, probability statements (pertaining to a population) are always true with reference to the represental; always false with reference to the particulars; and not relevant with reference to the universal.

2) One may define probability as the subjective degree of expectation (or belief) which arises out of our ignorance, and argue that the "probabilities are creation of the human mind" and "an omniscient who knows all the mechanisms of the universe in all details would need no probability" (Borel, 1965). Or in other words, an omniscient being has no need for representals. A little reflection would show that such an omniscient would not need universals either, because he knows each particular in all details. In the absence of universals there cannot be a language and hence his omniscience becomes non-communicable! May be the reason why God does not talk to us! Scientific knowledge being necessarily communicable, the representals are as much necessary and as much epistemologically legitimate, as the universals. Probabilities are based on human knowledge (at the intermediate level of cognition) and not on human ignorance. It is not correct to think that "probability assertions belong to a "myopic" view of the world" (Wittgenstein, 1967).

3) It is interesting to note that according to the Aristotelian two-valued logic: i) a particular entity can be either $A$ or not- $A$ (where $A$ is an attribute); ii) it cannot be neither $A$ nor not- $A$; and iii) it cannot be both $A$ and not- $A$. We may recall that: i) the state of parenthood of a particular man (like Mr. Jones) is either $C_{2}$ or not- $C_{2}$; ii) that of a man, the universal, is neither $C_{2}$ nor not- $C_{2}$; and iii) that of the represental man is both $C_{2}$ and not- $C_{2}$ (with respective probabilities). Thus the three specifications "eitheror", "neither-nor" and "both" pertain respectively to particulars, universals, and representals. The significance of this result deserves to be studied by pure philosophers, because the concept of universals has remained controversial from the days of Plato and the new concept of represental may play an important role at least in clarifying some of the issues.

\section{Concluding Remarks}

We have recognized that human knowledge is essentially based on three levels of cogni- 
tion which involve three epistemological concepts: particulars (objects of perception on which alone human experience and knowledge depend), universals (objects of thought which are necessary for forming general conclusions, for communication, and for expressing the laws of nature), and representals (objects of thought which have now become necessary for dealing with statistical data and atomic phenomena).

\section{Acknowledgements}

I am grateful to L. C. Mullatti and N. G. Mahadevappa for many helpful discussions, and to the Referee for his invaluable suggestions.

\section{References}

Borel, E. (1965). Elements of the Theory of Probability. New Jersey: Prentice-Hall.

Home, D., \& Whitaker, M. A. B. (1992). Ensemble Interpretations of Quantum Mechanics. A Modern Perspective. Physics Reports, 210, 224.

http://dx.doi.org/10.1016/0370-1573(92)90088-H

Howson, C. (1995). Theories of Probability. British Journal for Philosophy of Science, 46, 1-32. http://dx.doi.org/10.1093/bjps/46.1.1

Jaynes, E. (2003). Probability Theory: The Logic of Science. Cambridge: Cambridge University Press. http://dx.doi.org/10.1017/CBO9780511790423

Kendall, M. G. (1979). The History of Statistical Menthod. In W. H. Kruskal, \& J. M. Tanur, Eds., International Encyclopedia of Statistics. The Free Press.

Moreland, J. P. (2001). Universal. Acumen: McGill-Queen's University Press.

Quetelet, A. (1869). Physique Sociale ou sur le Development des Faculties de L'homme. Brussels.

Russell, B. (1948). Human Knowledge, Ch VI. London: Allen and Unwin.

Spigel, M. (1962). Theory and Problems of Probability and Statistics. New York: McGraw-Hill.

Umakantha, N. (2016). A New Approach to Probability Theory with Reference to Statistics and Statistical Physics. Journal of Modern Physics, 7, 989-1003.

http://dx.doi.org/10.4236/jmp.2016.79090

Wittgenstein, L. (1967). Probability. In P. Edwards, Ed., The Encyclopedia of Philisophy (Vol. 7, p. 468). New York: Macmillon and Free Press. 
Submit or recommend next manuscript to SCIRP and we will provide best service for you:

Accepting pre-submission inquiries through Email, Facebook, LinkedIn, Twitter, etc. A wide selection of journals (inclusive of 9 subjects, more than 200 journals)

Providing 24-hour high-quality service

User-friendly online submission system

Fair and swift peer-review system

Efficient typesetting and proofreading procedure

Display of the result of downloads and visits, as well as the number of cited articles

Maximum dissemination of your research work

Submit your manuscript at: http://papersubmission.scirp.org/

Or contact ojpp@scirp.org 